\title{
Mikrophysiologisches Retinamodell als Alternative zu Tiermodellen
}

\author{
JOHANNA CHUCHUY ${ }^{1,2}$, KEVIN ACHBERGER ${ }^{1}$, STEFAN LIEBAU ${ }^{1}$, PETER LOSKILL ${ }^{1,2}$ \\ ${ }^{1}$ MEDIZINISCHE FAKULTÄT, UNIVERSITÄT TÜBINGEN \\ 2 FRAUNHOFER-INSTITUT FÜR GRENZFLÄCHEN- UND BIOVERFAHRENSTECHNIK IGB, \\ STUTTGART
}

The visual system is one of the most important human senses. Hence, blindness leads to an unimaginable reduction of life quality. However, most diseases leading to the loss of vision are still incurable, because current models used in ophthalmic research fail to recapitulate the human eye in many crucial aspects. Combining human retinal organoids with Organ-on-a-chip technology may lead to a paradigm shift in the way we study pathogenesis and treatment of retinal diseases.

DOI: $10.1007 / \mathrm{s} 12268-020-1413-3$

(C) Die Autoren 2020

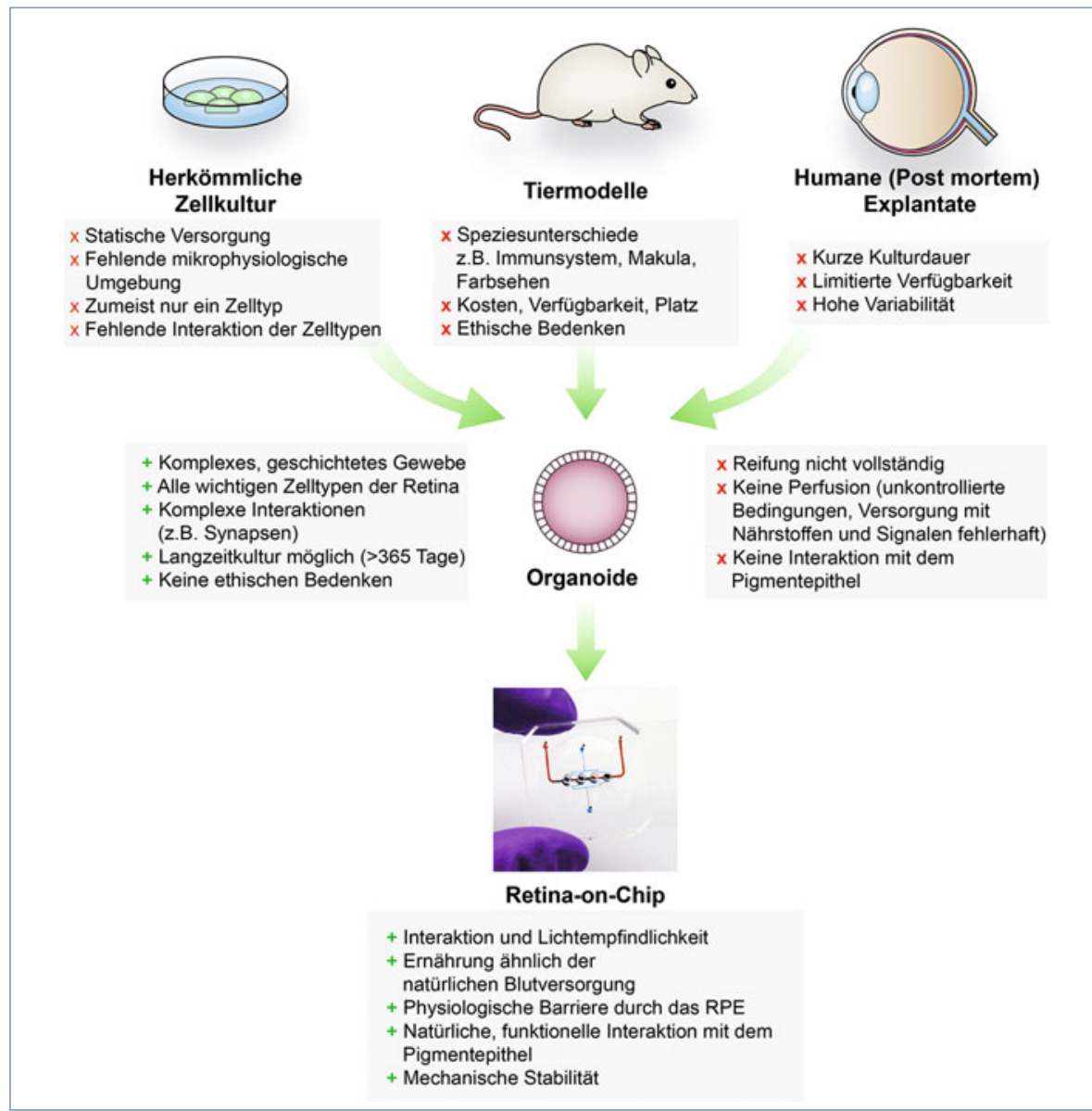

$\Delta$ Abb. 1: Vergleich bisheriger in vivo- und in vitro-Retinamodelle mit dem neuartigen Retina-onChip. RPE: retinales Pigmentepithel. Reproduziert aus [10], CC BY 4.0, aus dem Original übersetzt.
Das Auge mit der lichtempfindlichen Netzhaut (Retina) ist eines der wichtigsten Sinnesorgane des Menschen. Sehbeeinträchtigte oder gar erblindete Menschen leiden dementsprechend stark unter den Einschränkungen, die sich dadurch im Alltag ergeben. Nichtsdestotrotz gibt es bis heute weder eine Heilung noch eine wirksame Behandlung für eine Vielzahl verheerender Krankheiten, die häufig zum vollständigen Verlust der Sehkraft führen können, wie beispielsweise die diabetische Retinopathie oder Makuladegeneration. Ein wesentlicher Grund für die limitierten Therapieoptionen ist der Mangel an geeigneten Modellsystemen, welche das Verständnis über die zugrunde liegenden Krankheitsmechanismen und die Entwicklung von entsprechenden Medikamenten vorantreiben könnten (Abb. 1). Da es sich bei der Retina um ein extrem komplexes neuronales Gewebe handelt, welches durch bis zu sieben horizontal und vertikal verbundene Zelltypen vernetzt ist, lässt sie sich mit konventionellen in vitroZellassays (ein bis zwei unterschiedliche Zelltypen) nur unzureichend abbilden. Derzeit werden daher für solche Studien meist Tiermodelle wie Maus, Ratte oder Kaninchen herangezogen. Neben ethischen Bedenken besteht hier auch das Problem, dass die Tiere teils stark von der Anatomie und Biologie des Menschen abweichen. So besitzen die am häufigsten untersuchten Versuchstiere, Mäuse, keine Makula (Ort des schärfsten Sehens) und verfügen über ein weniger diverses Farbsehen. Darüber hinaus entwickeln Tiere oftmals nicht dieselben Krankheiten oder dieselbe Symptomatik, wie es beim Menschen der Fall ist (wie beispielsweise bei der altersbedingten Makuladegeneration). Infolgedessen können die aus Tiermodellen gewonnenen Erkenntnisse meist nicht oder nur eingeschränkt auf den Menschen übertragen werden, und entwickelte Therapien scheitern nicht selten nach jahrelangen und teuren Studien in den klinischen Phasen. 


\section{Mit neuen Technologien zum Tierversuchsersatz}

Eine mögliche Alternative bietet die Nutzung von in vitro-Gewebekulturen. In den letzten Jahren wurde eine neue Möglichkeit zur Generierung von dreidimensionalen in vitro-Modellen entdeckt: selbstformende Retina-Organoide [1, 2]. Diese Organoide, also Organ-ähnliche Strukturen, werden aus humanen induzierten pluripotenten Stammzellen (iPS) gewonnen und sind somit menschlichen Ursprungs. Die zugrunde liegenden iPS-Zellen können einfach aus Blut- oder menschlichen Haarzellen erzeugt werden $[3,4]$. Retina-Organoide bieten von den Ganglienzellen im Inneren bis hin zu einer Hülle aus Photorezeptoren ein physiologisches Gewebe mit anatomisch korrekter Anordnung. Somit besitzen diese Organoide fast alle relevanten Zellen zur Nachstellung der humanen Retina und sind sogar in der Lage, auf Lichtreize mit elektrischen Signalen zu reagieren [5]. Trotzdem fehlen den Retina-Organoiden einige wichtige physiologische Aspekte: So fehlt das retinale Pigmentepithel (RPE), das unter anderem notwendig ist, um einen funktionierenden Metabolismus der Photorezeptoren zu gewährleisten. Außerdem fehlt diesen Organoid-Systemen eine kontinuierliche Nährstoffversorgung, wie sie im Menschen durch den Blutkreislauf erfolgt, sowie wichtige Komponenten des Immunsystems.

Diese Nachteile können durch die Organon-a-chip-Technologie überwunden werden: Organ-on-a-chip-Systeme haben in den letzten Jahren immer mehr Aufmerksamkeit erweckt, da mit dieser Technologie kleinste Gewebeeinheiten in einer mikrophysiologischen Umgebung kultiviert und OrganLevel-Funktionalitäten nachgebildet werden können. Die Gewebe werden dabei über einen kontinuierlichen Medienfluss durch kleine Kanäle versorgt, die die menschlichen Blutgefäße nachbilden. Barriereschichten, ähnlich der Endothelbarriere, verhindern dabei zumeist, dass die Gewebe unphysiologischen Scherkräften ausgesetzt sind. Derartige Systeme existierten bereits für eine Vielzahl komplexer Organsysteme, wie Lunge [6], Herz [7], Fettgewebe [8] und Leber [9], nicht jedoch für die Retina. Durch Kombination der zwei zukunftsträchtigen Technologien - Organoide und Organ-on- $a$ chip - konnten wir den neuartigen Retinaon-Chip (RoC) entwickeln [10]. In einer mikrofluidischen Plattform werden Retina-
Organoide zusammen mit RPE-Zellen so integriert, dass dadurch ein komplexes Gewebemodell aus über sieben verschiedenen Zelltypen entsteht, das zugleich in eine mikrophysiologische Umgebung eingebettet ist.

\section{Das Retina-on-Chip-Modell}

Das Retina-on-Chip-Modell ist folgendermaBen aufgebaut (Abb. 2): In der untersten Lage des Chips befindet sich ein Blutgefäßähnlicher Kanal, der die Versorgung der darüberliegenden Strukturen über einen kontinuierlichen Medienfluss gewährleistet. Vier Gewebekammern ermöglichen es, bis zu vier Proben auf einem Chip zu kultivieren. Die Kammern sind dabei vom Kanal durch eine poröse Membran getrennt, welche die Aufgabe besitzt, einerseits die Zellen vor direkten Scherkräften des Medienflusses zu schützen und andererseits wichtige Nährstoffe zu den Zellen durchzulassen. Die Zellen werden über nach oben offene Kammern in das System gegeben. Zunächst werden die RPE-Zellen auf der Membran ausgesiedelt. Nachdem die Zellen nach ein bis zwei Tagen eine konfluente (homogene) Lage gebildet haben, wird im nächsten Schritt jeweils ein Retina-Organoid in jede der Kammern gegeben und mithilfe eines Hydrogels fixiert. Das Hydrogel bildet hierbei die physiologische extrazelluläre Matrix (EZM) nach. Zur Versorgung wird Kulturmedium mittels eines Pumpensystems kontinuierlich eingespeist. Mithilfe des RoC lässt sich so ein physiologisch relevantes Retinagewebe aufbauen, das alle wichtigen Zelltypen aufweist - einschließlich des wichtigen engen Kontakts von Photorezeptoren zum RPE. Das RPE ist unter anderem für die lebenslange Phagocytose („Verdauung“) von Außensegmenten der Photorezeptoren zuständig und stellt eine wichtige, sehr spezifische Barriere dar, die gleichzeitig eine kontinuierliche Versorgung von wichtigen metabolischen Ausgangsstoffen (VitaminA-Derivaten) gewährleistet.

\section{Retina-on-Chip kann komplexe zelluläre Kreisläufe imitieren}

Um zu untersuchen, ob abgesehen von der anatomisch korrekten Anordnung der Zelltypen auch die Funktionalität der Zellen gewährleistet ist und zelluläre Kreisläufe, wie z. B. die eben beschriebene Phagocytose, im Chip nachgestellt werden können, wurden die Photorezeptoren der Organoide

\section{Hier steht eine Anzeige.}

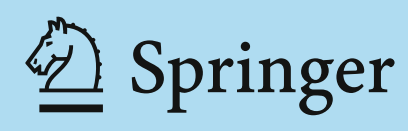



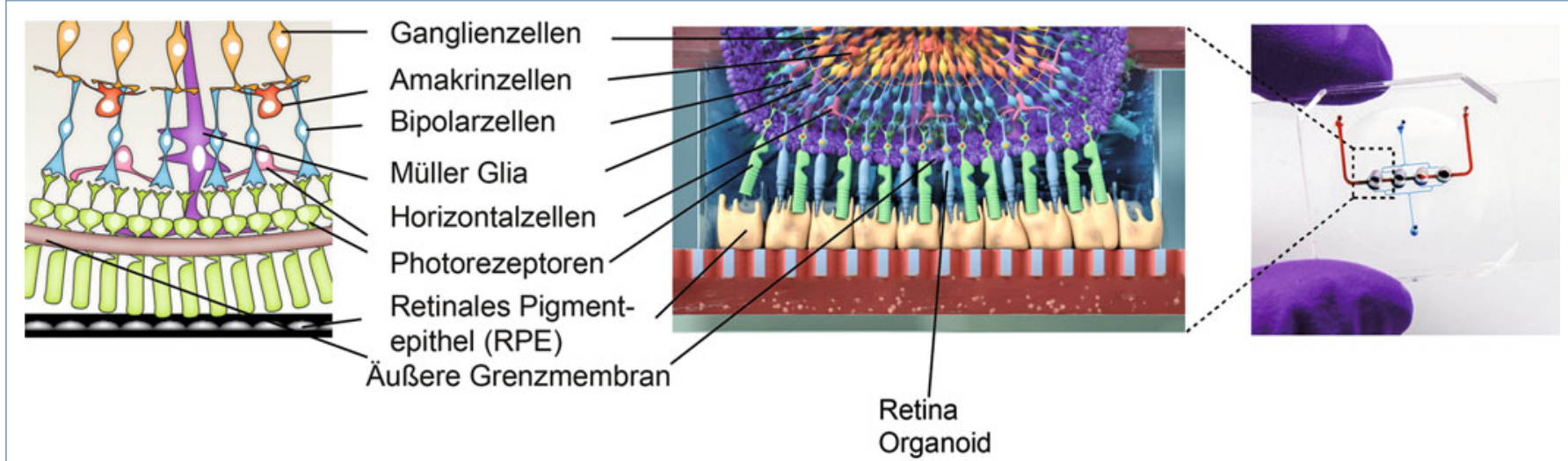

$\Delta$ Abb. 2: Retina-on-Chip. Schematischer Vergleich des Aufbaus der menschlichen Retina (links) mit dem Retina-on-Chip (Mitte). Rechts: fotografische Abbildung des Retina-on-Chip. Die mikrofluidischen Kanäle sind zur Verdeutlichung mit Farbstoffen (rot und blau) angefärbt. Reproduziert aus [9], CC BY 4.0, aus dem Original übersetzt.

sowie die RPE-Zellen mit unterschiedlichen Farbstoffen angefärbt. Somit ist es möglich, die Interaktion der Zellarten in Echtzeit zu verfolgen (Abb. 3).

Durch Echtzeit-Mikroskopie konnte gezeigt werden, dass sich bereits nach wenigen Tagen Teile des Farbstoffs der Photorezeptoren in den RPE-Zellen wiederfinden, was darauf schließen lässt, dass das RPE den Aufnahme- (Phagocytose) bzw. RecyclingProzess erfüllt und damit die Funktionalität des Modells bestätigt.

\section{Anwendungsmöglichkeiten}

Das RoC-Modell bietet eine Vielzahl von Anwendungsmöglichkeiten, sowohl als Testsystem für die pharmazeutische Industrie als auch als Krankheitsmodell für die biomedizinische Grundlagenforschung. Um die Eignung des Systems zur Medikamentenuntersuchung festzustellen, wurden bereits Substanzen untersucht, deren schädliche Nebenwirkungen auf die Retina des Menschen bereits bekannt ist. Dabei konnte nicht nur gezeigt werden, dass unterschiedliche Konzentrationen der Medikamente im Vergleich zu Kontrollen ohne Medikament dosisabhängig zum Zelltod führten, sondern auch, dass die physiologische Struktur dazu führt, dass die einzelnen Zellen vor dem Medikament deutlich geschützt sind. Insgesamt lässt sich schlussfolgern, dass der RoC geeignet ist, um Untersuchungen von Substanzen auf die neuralen Zellen der Netzhaut durchzuführen.

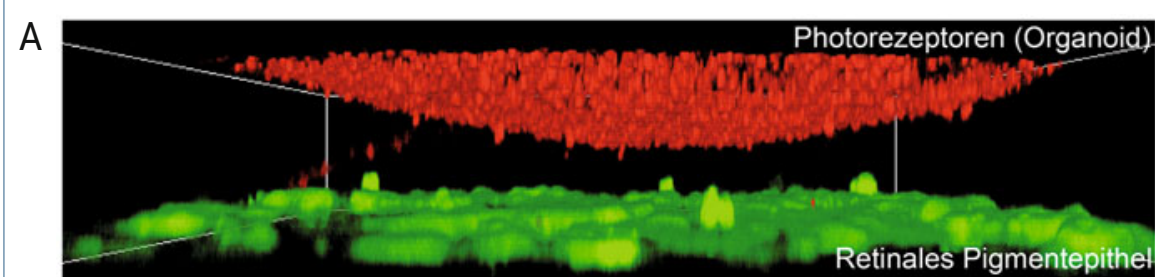

B
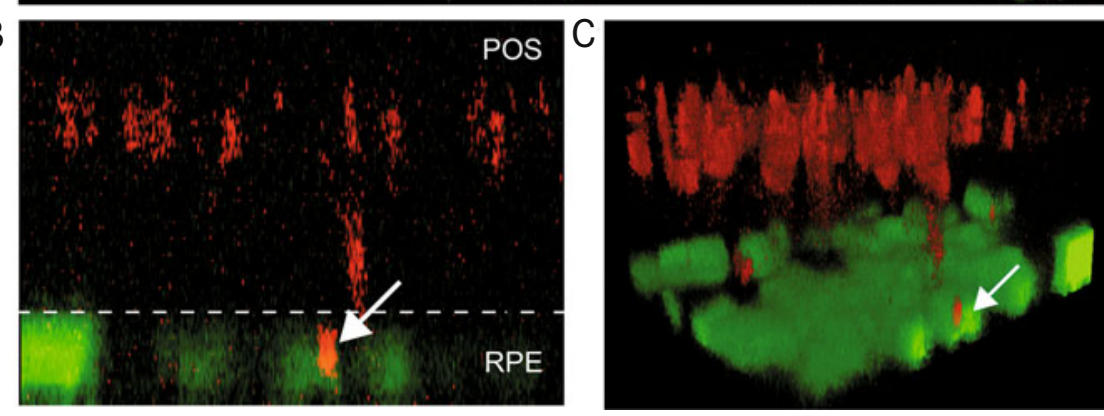

$\triangle$ Abb. 3: Phagocytose von Photorezeptorsegmenten (POS) durch das retinale Pigmentepithel (RPE) im Chip. A, 3D-Darstellung eines fluoreszenzmarkierten Retina-Organoids (rot) und retinalen Pigmentepithels (grün). 2D- (B) bzw. 3D-Darstellung (C) der Phagocytose von AußensegmentanteiIen (Pfeil) im RPE. B, C reproduziert aus [10], CC BY 4.0.

\section{Ausblick: der Retina-Chip als Plattformtechnologie}

Die Vereinigung von Stammzell-Organoidund Organ-on-a-chip-Technologie erlaubt es, das System zukünftig um weitere komplexe Aspekte zu vervollständigen. So ist es möglich, weitere Zellarten oder gar OrganoidZellverbände aus (denselben) Stammzellen zu generieren und diese modular in das Chipsystem zu integrieren. Konkret könnten beispielsweise Endothelzellen (Blutgefäße), retinaspezifische Immunzellen (Mikroglia) oder gar Gehirn-Organoide (brain organoids) zur neuralen Verschaltung der Retina vernetzt werden. Solche Systeme könnten die Komplexität und Physiologie des menschlichen Auges in einem bisher unerreichten Ausmaß simulieren. Darüber hinaus ist es möglich, den Retina-on-Chip in ein „MultiOrgan-Chip“-Konzept zu integrieren. Dies erlaubt es, mehrere Organe mit einer Art künstlichem Kreislauf miteinander zu verschalten (z. B. in Form von einer Kombination aus Leber, Fettgewebe und Netzhaut), um so die komplexen Stoffwechselinteraktionen der Gewebetypen zu simulieren. Der Retina-on-Chip ist damit ein Baustein, der die ophthalmologische Forschung ein gutes Stück näherbringt, Tierversuche zu minimieren oder gar zu ersetzen, und könnte in der Zukunft dazu beitragen, Medikamente schneller und zuverlässiger in die klinische Phase und zum Patienten zu bringen.

\section{Literatur}

[1] Eiraku M, Takata N, Ishibashi H et al. (2011) Selforganizing optic-cup morphogenesis in three-dimensional culture. Nature 472: 51-56

[2] Nakano T, Ando S, Takata N et al. (2012) Self-formation of optic cups and storable stratified neural retina from human ESCs. Cell Stem Cell 10:771-785 
[3] Loh Y-H, Agarwal S, Park I-H et al. (2009) Generation of induced pluripotent stem cells from human blood. Blood 113:5476-5479

[4] Aasen T, Raya A, Barrero MJ et al. (2008) Efficient and rapid generation of induced pluripotent stem cells from human keratinocytes. Nat Biotechnol 26:1276-1284 [5] Zhong X, Gutierrez C, Xue T et al. (2014) Generation of three-dimensional retinal tissue with functional photoreceptors from human iPSCs. Nat Commun 5:4047 [6] Huh D, Matthews BD, Mammoto A et al. (2010) Reconstituting organ-level lung functions on a chip. Science 328:1662-1668

[7] Schneider O, Zeifang L, Fuchs S et al. (2019) Userfriendly and parallelized generation of human induced pluripotent stem cell-derived microtissues in a centrifugal heart-on-a-chip. Tissue Eng Part A 25:786-798

[8] Rogal J, Binder C, Kromidas E et al. (2020) WAT-on-achip integrating human mature white adipocytes for mechanistic research and pharmaceutical applications. Sci Rep $10: 6666$

[9] Bhise NS, Manoharan V, Massa S et al. (2016) A liveron-a-chip platform with bioprinted hepatic spheroids. Biofabrication 8:014101

[10] Achberger K, Probst C, Haderspeck JC et al. (2019) Merging organoid and organ-on-a-chip technology to generate complex multi-layer tissue models in a human retinaon-a-chip platform. Elife 8:e46188
Funding: Open Access funding provided by Projekt DEAL.

Open Access: Dieser Artikel wird unter der Creative Commons

Namensnennung 4.0 International Lizenz veröffentlicht, welche die Nutzun ervielfältigung, Bearbeitung, Verbreitung und Wiedergabe in jeglichem Medium und Format erlaubt, sofern Sie den/die ursprünglichen Autor(en) und beifügen und angeben, ob Änderungen vorgenommen wurden. Die in diesem Artikel enthaltenen Bilder und sonstiges Drittmaterial unterliegen ebenfalls der genannten Creative Commons Lizenz, sofern sich aus der Abbildungslegende nichts anderes ergibt. Sofern das betreffende Material nicht unter der genannten Creative Commons Lizenz steht und die betreffende Handlung nicht nach gesetzlichen Vorschriften erlaubt ist, ist für die oben aufgeführten Weiterverwendungen des Materials die Einwilligung entrehmen Sie bitte der Lizenzinformation auf http// creativecommons.org/ /by/4.0/deed.de.

\section{Korrespondenzadresse:}

Jun.-Prof. Dr. Peter Loskill

Forschungsinstitut für Frauengesundheit (FFG)

Eberhard Karls Universität Tübingen

Calwerstraße 7

D-72076 Tübingen

peter.loskill@uni-tuebingen.de

\section{AUTOREN}

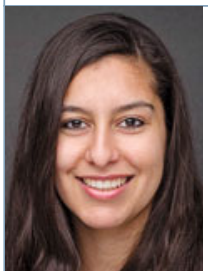

Johanna Chuchuy

Jahrgang 1989. Bachelor und Master in Maschinenbau an der Universität Stutt-

gart. Seit 2018 Promotionsstudium an den Universitäten Tübingen und Stuttgart.

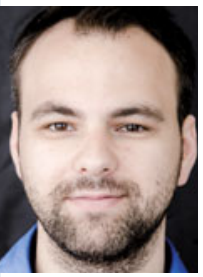

Kevin Achberger

Jahrgang 1989. Bachelor und Master in Molekulare Medizin in Ulm. 2019 Promo-

tion (Dr. rer. nat.) an der Universität Tübingen; dort seit 2019 Postdoc am Institut für Neuroanatomie und Entwicklungsbiologie.

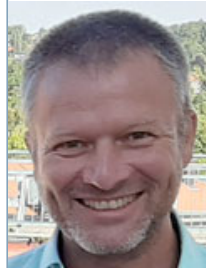

\section{Stefan Liebau}

Jahrgang 1973. Humanmedizinstudium an der Universität UIm; dort 2004 Promotion, 2004-2013 Postdoc am Institut für Anatomie und Zellbiologie. Seit 2013

Direktor des Instituts für Neuroanatomie und Entwicklungsbiologie der Universität Tübingen.

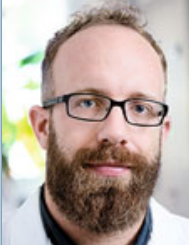

Peter Loskill

Jahrgang 1984. Physikstudium an der Universität des Saarlandes, dort 2012 Promotion. 2013-2015 Postdoc an der University of California, Berkeley, USA. 2016-2019 Attract-Gruppenleiter am Fraunhofer-Institut für Grenzflächen- und Bioverfahrenstechnik IGB, Stuttgart. Seit 2018 Juniorprofessor für Experimentelle Regenerative Medizin an der Universität Tübingen. Seit 2019 Leiter des Innovationsfeldes Zell- und Gewebetechnologien am Fraunhofer IGB.

\section{Hier steht eine Anzeige.}

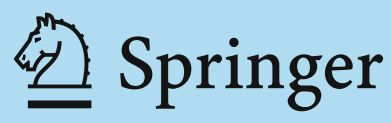

